pulmonary arterial hypertension (PAH) cases and approximately $25 \%$ of idiopathic PAH cases. PAH may arise due to endothelial dysfunction as mice with BMPR-II deficiency exhibit increased pulmonary vascular permeability.

BMP9 is an endothelial quiescence factor and is thought to maintain the integrity of the endothelium. We previously reported that BMPR-II and ALK1 are the key receptors through which BMP9 inhibits the proliferation of human pulmonary artery endothelial cells (hPAECs). We hypothesised that BMPR-II deficiency impacts on endothelial cell connectivity and may contribute to endothelial dysfunction in PAH.

Methods Human pulmonary artery endothelial cells were obtained from Lonza and blood outgrowth endothelial cells (BOECs) were isolated from peripheral blood of unaffected controls or PAH patients with identified BMPR-II mutations. Cells were transfected with siRNAs targeting BMPR-II followed by stimulation with BMP9. RNA was extracted and the expression of candidate genes determined by quantitative PCR. Further siRNA studies were performed for ALK1 and endoglin siRNAs. The promotion of gap junction assembly by BMP9 and BMP10 were assessed by immunofluorescence, Western blotting and functionally using parachute assays.

Results Screening of candidate BMP9-induced junctional and structural proteins highlighted a subset of endothelial connexins that are BMP9 and BMP10-responsive and dependent on BMPR-II and ALK1. BMP9 and BMP10 increased the expression of the connexins, assessed by Western blotting and immunostaining. In addition, BMP9 and BMP10 significantly increased the transfer of calcein from labelled donor cells to unlabelled acceptor cells, indicating a promotion of endothelial cell connectivity.

Conclusion In addition to their roles promoting endothelial quiescence, BMP9 and BMP10 directly promote endothelial cell connectivity by increasing connexin expression and assembly. The central contributions of BMPR-II and ALK1 to this process may implicate impaired endothelial connectivity as a pathological component of PAH and HHT.

\section{S36 FERROPORTIN IS EXPRESSED IN HUMAN PULMONARY ARTERY SMOOTH MUSCLE CELLS: IMPLICATIONS FOR PULMONARY ARTERIAL HYPERTENSION}

L Ramakrishnan, S Mumby, JS Wort, G Quinlan. Imperial College London, London, UK

\subsection{6/thoraxjnl-2014-206260.42}

Background Pulmonary Arterial Hypertension (PAH) is a rare but fatal condition manifested by pulmonary vascular remodelling, increased pulmonary vascular resistance and right-heart failure. Disruption in iron handling and anaemia, caused by elevated iron-regulatory hormone hepcidin, is observed in PAH. Ferroportin the only known cellular iron-export protein is downregulated by hepcidin. As such, iron supplementation as a therapy is currently under clinical trial. However, it is also known that iron is both pro-oxidant and pro-proliferative. Latest evidence also points to sub-clinical haemolysis and the presence of free haemoglobin in PAH patients. We hypothesised that ferroportin would be expressed; be responsive to hepcidin challenge and have implications for the proliferation of human pulmonary artery smooth cells (hPASMCs).

Methods The mRNA levels of ferroportin was measured by RT-PCR, the protein expression was detected by western-blot analysis and quantified by ELISA. The sub-cellular distribution of

\section{Change in ferroportin levels $24 \mathrm{~h}$ after hepcidin treatment in PASMCs}
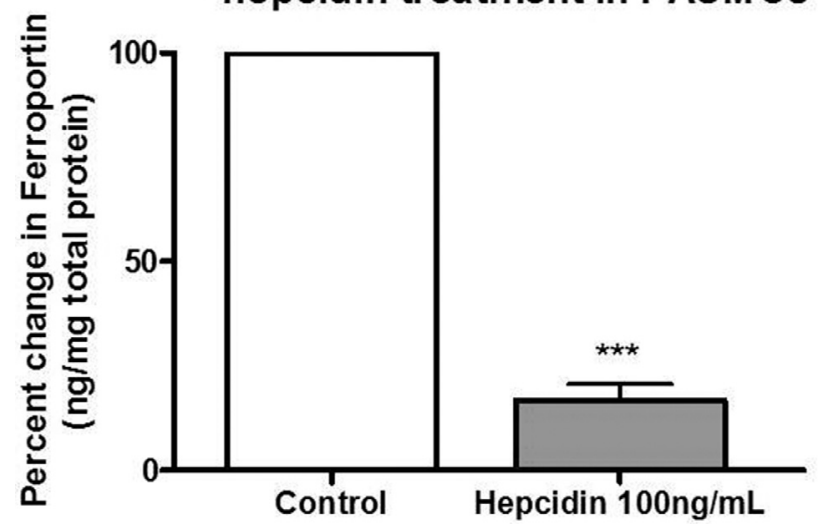

Abstract S36 Figure 1 Confluent PASMCs (passage 4-8) were treated with $100 \mathrm{ng} / \mathrm{ml}$ hepcidin for 22-24 h and total protein extracted using cell-lysis buffer. Ferroportin expression was quantitated using an ELISA kit and normalised to total protein estimated by bradford reagent. $n=4,{ }^{* *}=p<0.001$

ferroportin was visualised by immunocytochemistry (ICC). hPASMCs were pre-incubated with or without free haemoglobin and further challenged with increasing doses of hepcidin and the proliferative responses assessed by cyquant and/or BrdU incorporation assays. Some cells were also pre-incubated with LY2928057 (monoclonal antibody against ferroportin that stabilises cellular expression, Eli-Lily) in proliferation assays.

Results Basal ferroportin mRNA was detected in hPASMCs, but the mRNA levels were largely unaltered with hepcidin exposure $(\mathrm{n}=3)$. A $\sim 50 \mathrm{KDa}$ protein band representing ferroportin was detected under resting conditions while hepcidin challenge caused decrease in ferroportin protein levels (Figure 1). Basal ferroportin was uniformly distributed in the cells; however hepcidin treatment led to intense punctate/vesicular staining $(n=3)$. Finally, exposure to free haemoglobin alone or along with hepcidin increased proliferation of hPASMCs by $13.6 \%$ and $12.4 \%$ ( $p$ $<0.05, \mathrm{n}=3$ ) respectively. Interestingly, pre-incubation of the cells with LY2928057 partly reversed this effect.

Conclusion This is the first report of ferroportin expression and regulation in hPASMCs. We suggest that targeting and manipulating the hepcidin-ferroportin axis using LY2928057 might prove a novel therapeutic approach for PAH.

\section{S37 VASCULAR ENDOTHELIAL CELL GROWTH FACTOR-A (VEGF-A) SIGNALLING AND NEOVASCULARISATION OF PULMONARY ENDARTERECTOMY MATERIAL IN CHRONIC THROMBOEMBOLIC PULMONARY HYPERTENSION (CTEPH)}

M Southwood, C Hadinnapola, E Moseley, D Jenkins, M Goddard, K Sheares, M Toshner, J Pepke-Zaba. Papworth Hospital NHS Foundation Trust, Cambridge, UK

\subsection{6/thoraxjnl-2014-206260.43}

Background Despite recent advances in the medical treatment of patients with CTEPH, relatively little is understood surrounding the underlying pathological mechanisms. Many patients have a historical documented venous thromboembolic event (VTE) and consequently, failed resolution of an acute VTE has been proposed as a key initiating factor in the subsequent development of CTEPH. Here we investigated VEGF-A levels, a key regulator of angiogenesis, in CTEPH patients prior to and following 
pulmonary endarterectomy (PEA) surgery to remove the thromboembolic material. We also examined the surgically excised PEA material and measured neovessel density in distal regions of the specimens.

Methods and results Serum VEGF-A levels were measured by Luminex array in paired serum samples from $n=44$ patients at baseline (before PEA surgery) and following PEA surgery. Following PEA surgery, serum VEGF-A levels were significantly reduced compared to baseline measurements (159.5 \pm 174.8 vs. $194.4 \pm 198.2, \mathrm{p}=0.0182)$. Distal regions of excised PEA material were cross sectioned and processed for histopathological examination. Neovessel density was calculated by counting the absolute number of vascular channels present within a cross-sectional area and normalised to tissue area using Image J. We also measured the time between documented VTE and subsequent PEA surgery (VTE-PEA time) in patients with a documented VTE ( $\mathrm{n}=40 / 44$ [90\%] patients), and identified an inverse correlation between neovessel density and VTE-PEA time.

Conclusions Extensive angiogenesis was evident in distal PEA material from patients with CTEPH. VEGF-A is known to be a key regulator of angiogenesis and serum levels were significantly reduced following PEA surgery. Interestingly, a significant inverse correlation between neovessel density and VTE-PEA time was observed indicating that in the early stages following VTE, extensive angiogenesis is evident within the thromboembolus. Taken together these data provide further evidence that angiogenesis is an important mechanism in the attempted resolution of a VTE.

\section{S38 THE BRD4 INHIBITOR, JQ1 DECREASES PROLIFERATION AND ARRESTS THE CELL CYCLE OF PULMONARY VASCULAR CELLS: IMPLICATIONS FOR PULMONARY} ARTERIAL HYPERTENSION

${ }^{1} \mathrm{~S}$ Mumby, ${ }^{2} \mathrm{~N}$ Gambaryan, ${ }^{2} \mathrm{~A}$ Adcock, ${ }^{1} \mathrm{~S} J$ Wort. ${ }^{1}$ Vascular Biology, National Heart and Lung Institute, Imperial College London, London, UK; ${ }^{2}$ Airway Disease, National Heart and Lung Institute, Imperial College London, London, UK

\subsection{6/thoraxinl-2014-206260.44}

Background Pulmonary arterial hypertension (PAH) is an incurable disease characterised by raised pulmonary resistance, resulting from vascular remodelling which leads to right heart failure and death. Recently NF-kB mediated inflammatory gene expression and vascular proliferation/remodelling have been shown to be involved in the pathogenesis of PAH. The expression of subsets of NF-kB-induced inflammatory genes is dependent upon the recruitment of the bromodomains and extra-terminal (BET) family of proteins to the transcriptional activation complex. We hypothesise that inhibition of BET proteins which bind acetylated lysine residues on histones and non-histone proteins will attenuate the hyperproliferative and proinflammatory phenotype of vascular cells.

Methods Primary human pulmonary vascular endothelial cells (P-EC) were serum starved for $24 \mathrm{~h}$ prior to treatment with the Brd4 mimic JQ1+ or JQ1- (inactive enantiomer) in complete (5\% FCS) media. P-EC cell proliferation was measured by BrdU incorporation and apoptosis was determined using caspase 3/7 activity. Cell cycle progression was determined by FACs analysis. mRNA levels of cell cycle genes and inflammatory cytokines were measured by RT-PCR. MTT assay was used to measure cell viability.

Results JQ1+ caused a significant $(\mathrm{p}<0.001)$ and concentration-dependent decrease in P-EC proliferation and an increase in caspase 3/7 activity compared to P-EC treated with JQ1- for 24 h. JQ1+ $(1 \mu \mathrm{M})$ significantly arrested the cell cycle of P-EC at the G1 phase. This was additionally evidenced by a decrease in the cell cycle genes CDK2, 4 and 6 mRNA levels and a significant increase in the mRNA of the cell cycle inhibitor CDKN1A (p21/cip1) at 4 h. Finally, JQ1+ significantly $(\mathrm{p}<0.01)$ inhibited the mRNA levels of the inflammatory cytokines IL-6 and 8 in P-EC compared to JQ1-.

Conclusion Inhibition of Brd4 with JQ1 decreases remodelling and inflammation in P-EC via a decrease in proliferation, cell cycle arrest and an increase in apoptosis. Further work is required but Brd4 inhibition may provide therapeutic drugs for the treatment of PAH.

\section{S39 THE ROLE OF SOLUBLE GUANYLATE CYCLASE STIMULATOR BAY 41-2272 ON REMODELLING PROCESSES RELEVANT TO THE PATHOGENESIS OF PULMONARY ARTERIAL HYPERTENSION}

D Shao, SJ Wort. Imperial College London, London, UK

\subsection{6/thoraxjnl-2014-206260.45}

Introduction and objectives Pulmonary arterial hypertension (PAH) is characterised by remodelling of small, muscular precapillary blood vessels. The subsequent rise in pulmonary vascular resistance leads to right ventricular failure and death. The aetiology of the remodelling process is largely unknown although defects in the bone morphogenetic protein receptor II (BMPR II) pathway are likely to be involved. Most of the therapies used thus far are aimed at pulmonary vasodilation. However it is unclear how much of the benefit seen with these medications is related to reverse remodelling. Riociguat is a "first in class" drug that stimulates soluble guanylate cyclase, with a consequent increase in cyclic GMP (and vasodilation). Riociguat has recently been shown to improve haemodynamics and exercise capacity in patients with idiopathic $\mathrm{PAH}$ and chronic thromboembolic PH (PATENT and CHEST). Here we sought to

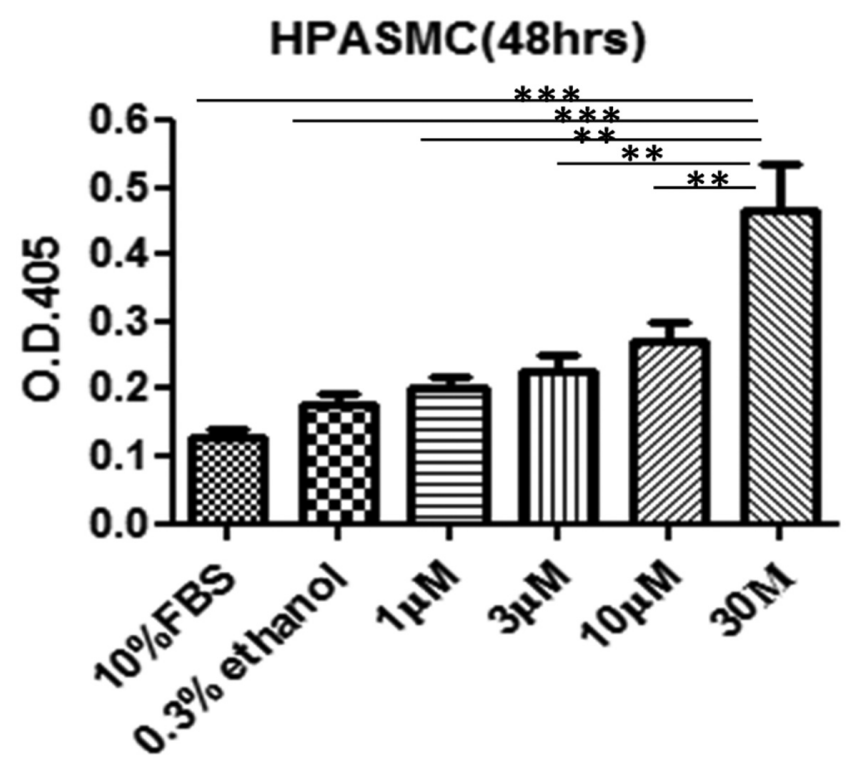

Abstract S39 Figure 1 Bay41-2272 induces HPASMCs DNA fragmentation (apoptosis), data were presented as mean \pm SEM $n=3$. ${ }^{* *} \mathrm{p}<0.005 ;{ }^{* *} \mathrm{p}<0.001$ 